\title{
EVALUATION OF BOND STRENGTH OF RESIN NANO-CERAMIC AS POST MATERIAL
}

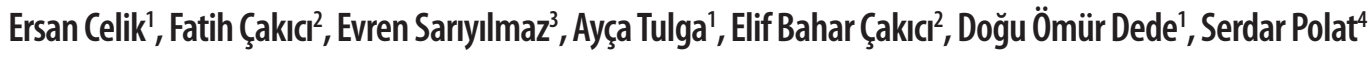 \\ 'Department of Prosthodontics, Faculty of Dentistry, Ordu University, Turkey \\ ${ }^{2}$ Department of Endodontics, Faculty of Dentistry, Ordu University, Turkey \\ ${ }^{3}$ Department of Endodontics, Faculty of Dentistry, Çanakkale Onsekiz Mart University, Turkey \\ ${ }^{4}$ Department of Prosthodontics, Faculty of Dentistry, Gazi University, Turkey
}

\begin{abstract}
INTRODUCTION: Different usage areas of newly developed restorative materials have increased..

ОвJеCTIVEs: The purpose of this study was to investigate usability of a newly produced resin nano-ceramic (RNC) as a post material, and how to increase bond strength of different post systems to root canal dentin.

MATERIAL AND METHODS: Crowns of eighty extracted maxillary central incisors were separated transversally at cementoenamel junction. The specimens were then endodontically prepared. Post spaces were opened at a diameter of $1.8 \mathrm{~mm}$ with a length of $10 \mathrm{~mm}$ on the root, maintaining $4 \mathrm{~mm}$ of filling material in apical third. $40 \mathrm{RNC}$ post and 40 fiber post samples were divided into 4 sub-groups $(n=10)$, and different surface treatments were divided into control (Cnt), tribochemical coating (Tbc), and laser-irradiated (Lsr 1 and Lsr 2) groups. After adhesive cementation of posts to root canal dentin, the specimens were cut with $1 \mathrm{~mm}$ thicknesses. Push-out bond strength (PBS) tests were performed using a universal testing machine, and the results were analyzed using two-way analysis of variance and Tukey tests.

RESULTS: The highest PBS value for fiber post groups was observed in the Tbc group (6.51 MPa). The highest PBS values were observed in the Tbc group (6.22 MPa), particularly in the RNC post group, and this value was statistically different from the other groups $(p<0.05)$.

ConcLusions: Tbc treatment of posts may be a suitable way for increasing the bond strength. The experimentally produced RNC posts showed PBS values close to the prefabricated fiber posts. Therefore, they could be an alternative restorative material for repairing endodontically treated teeth.
\end{abstract}

KEY WORDS: surface treatment, fiber post, resin nano-ceramic.

\section{INTRODUCTION}

For many years now, post core restorations are used for restoring endodontically treated teeth with excessive loss of crown structure. Due to some disadvantages, casted metal post restorations have been almost completely abandoned and replaced by current post systems.
Different post materials have been developed in parallel with developments in material technology. The greatest advantages of these materials are that they have an elastic modulus close to elastic modulus of the dentin, therefore, they prevent roots' fractures. In addition, they present aesthetic advantages, especially under all-ceramic restorations [1].

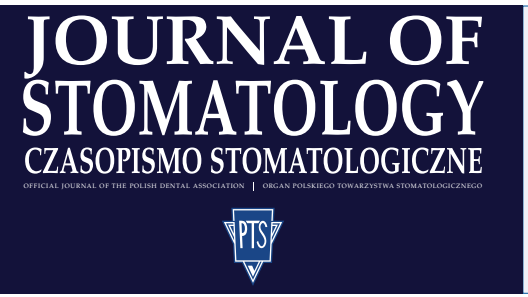

AdDRESS FOR CORRESPONDENCE: Dr. Ersan Celik,

Department of Prosthodontics, Ordu University Faculty of Dentistry, Cumhuriyet Campus, Mustafa Kemal Street, No: 254, 52200 - Altınordu, Ordu, Turkey, e-mail: ersancelik@gmail.com

ReCEIVED: 12.03.2021 • ACCEPTED: 19.04.2021 • PUBlished: 04.06.2021 
Current post materials include fiber posts, which have become very popular in recent years. Fiber posts were firstly made by Dure in 1990. Fredriksson et al. investigated the survival of restored teeth with fiber posts in 236 patients, and reported that only 5 teeth failed at the end of 3 years [2]. Nowadays, hybrid ceramics have been introduced and provide better physical, mechanical, and enhanced flexural properties of ceramics and composites [3]. However, the major disadvantage of these post systems is the uncertainty about the adhesion to root canal dentin. There is no recent literature on the use of hybrid ceramics in this field. Therefore, several methods have been developed to increase the bonding between the two structures. Blasting and acid etchings are among these methods $[3,4]$.

The development in laser technology increased the use of this device in the dental sector. In endodontic treatments, laser is usually used to remove organic and inorganic tissues within the root canal, and this technique significantly reduce bacterial contamination. The use of laser to reduce bacterial contamination along the root canal changes the dentin surface. The dentin surface is an important feature for the cementation of intra-radicular posts $[5,6]$. At the same time, surface of the post material affects the bond strength to the root canal dentin. For this reason, dental lasers are also used to roughen the surface of restorative materials $[3,4]$. Nd: YAG (neodymium-doped: yttrium, aluminum, garnet), Er: YAG (erbium: yttrium, aluminum, garnet), and Er, Cr: YSGG (erbium, chromium: yttrium, scandium, gallium, garnet) lasers have been used as an alternative surface treatment methods of dental materials in dentistry. The term 'ablation', which is the working principle of lasers, is the mechanism of removing the particles from the surface of materials by micro explosion and vaporization. Therefore, this process provides an increase in micromechanical retention $[3,6]$.

Self-adhesive cement materials have been introduced to decrease the number of cementation steps by eliminating a pre-treatment of tooth. The adhesion results from acidic monomers that are simultaneously mineralized into lower layers of the tooth, which cause micromechanical retention. Furthermore, secondary re-

\section{TABLE 1. Surface treatments groups}

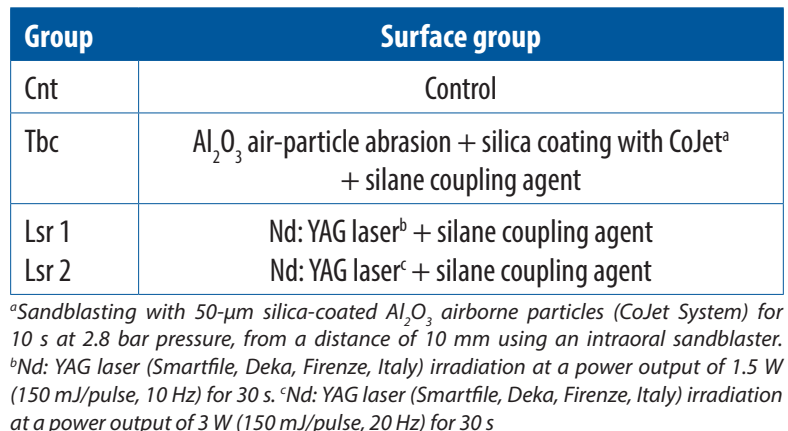

actions produce additional chemical bonds to the dentin surface [7].

\section{OBJECTIVES}

The modification of the post surface directly affects the bond strength of the cement in the root canal and there are limited studies on the effect of high-density lasers on the bond strength of self-adhesive adhesion materials. The aim of this study was to investigate the usability of a newly produced resin nano-ceramic as a post material, and how to increase the bond strength of different post systems to the root canal dentin. The first null hypothesis of the study was that the bond strength to the root canal dentine would not vary depending on the type of post materials. The second null hypothesis was that different surface treatments would not affect the bond strength of post materials.

\section{MATERIAL AND METHODS}

This study was approved by ethics committee of the Ordu University (2015/13).

Eighty non-carious extracted human maxillary central incisors with the same root lengths, uncracked, without restorations were selected for the study. The crown sections of the teeth were separated horizontally at the cemento-enamel junction with a diamond disc using a precious cutting machine (Mecatome T180, Presi, Eybens, France) under water cooling.

The specimens were then endodontically prepared with crown-down technique as in a previous study [8]. The working length was set to $1 \mathrm{~mm}$ short from apical foramen of the root. The irrigation was made by $2 \mathrm{ml}$ of $2.5 \%$ sodium hypochlorite. Subsequently, the root canals were filled with AH Plus sealer (Dentsply DeTrey, Konstanz, Germany) and gutta-percha cones (Dentsply Maillefer, Ballaigues, Switzerland). The samples were stored in an incubator (Memmert IN 75, Memmert GmbH, Schwabach, Germany) at $37^{\circ} \mathrm{C}$ and $100 \%$ relative humidity for 24 hours. The next day, the gutta-percha cones were removed by using fiber post-drills (Cytec Blanco, Hahnenkratt, Königsbach-Stein, Germany). Post space was opened by a bur compatible for the fiber post at a diameter of $1.8 \mathrm{~mm}$ and with a length of $10 \mathrm{~mm}$ on the root, maintaining $4 \mathrm{~mm}$ of filling material in the apical third. Then it was washed with $17 \%$ EDTA solution and distilled water, and dried with paper points. For the preparation of RNC posts, a $1.8 \mathrm{~mm}$ diameter fiber post was scanned with an extraoral scanner (3Shape D700, 3Shape, Copenhagen, Denmark) and 40 copies were made from RNC blocks (LAVA Ultimate, 3M Espe, St. Paul, Minnesota, USA) using CAD-CAM (Yenamak D50, Yenadent Ltd., Istanbul, Turkey) procedure.

40 RNC ceramic post and 40 fiber post samples were divided into 4 sub-groups $(n=10)$. Posts in sub-groups were exposed to the surface treatments specified in Table 1. 


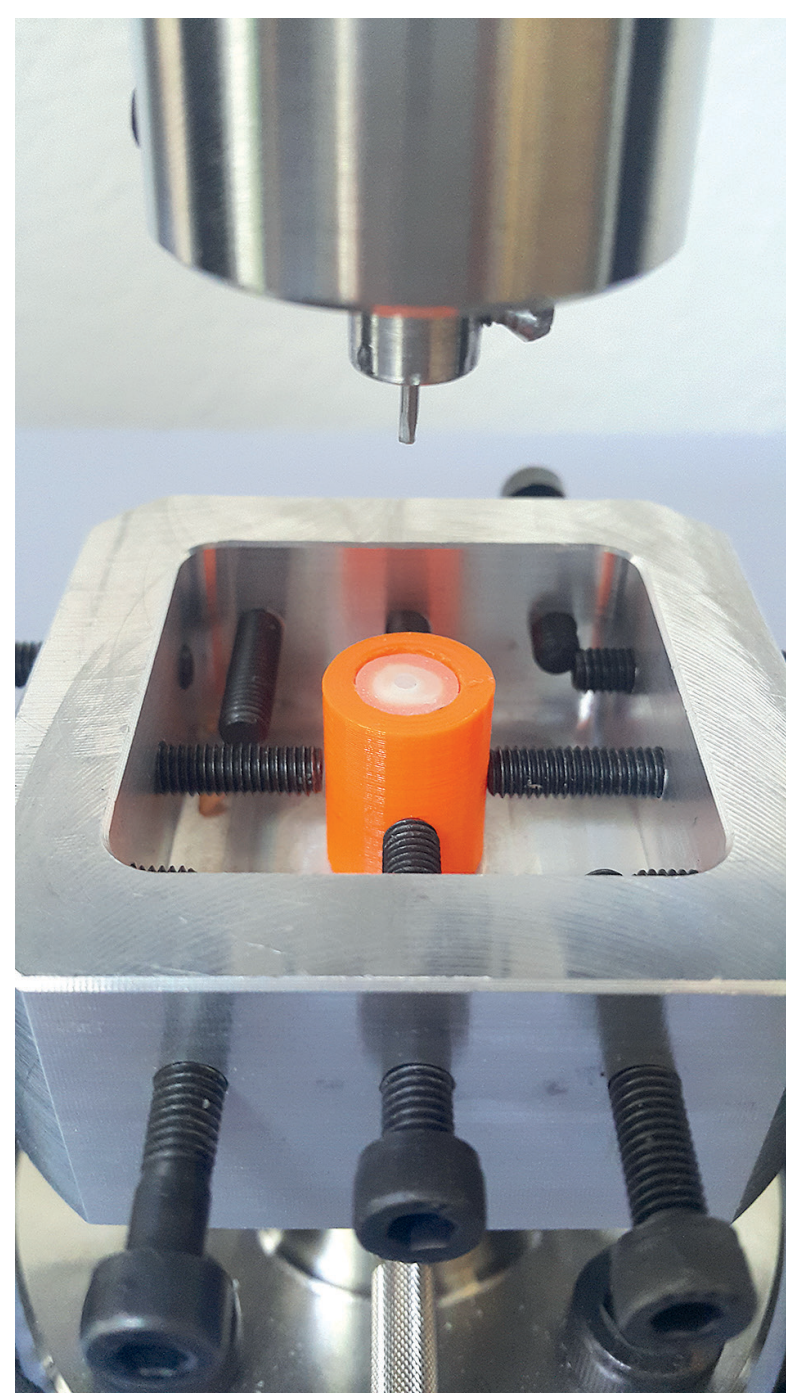

FIGURE 1. Specimen in the universal testing machine

For the cementation, self-adhesive resin cement ( $\mathrm{Pa}$ navia SA Cement, Kuraray, Chiyoda, Tokyo, Japan) was sent to post cavity and also smeared around the post. Then, the posts were inserted into the root canals. All samples were activated with a total of $60 \mathrm{sec}$ light from buccal and lingual surfaces. Each tooth was then embedded in an autopolymerising acrylic resin (ShangChi, Shanghai Medical Instruments Co., Ltd., Shanghai, China) with a surveyor (APF450, Amann-Girrbach, Koblach, Austria). Each root was segmented opposite to its long axis to make examples at $1 \mathrm{~mm}$ thickness at the coronal third of the root. One part of the $1 \mathrm{~mm}$ thick example was set up from every tooth.

Push-out bond strength was tried using a universal testing machine (AGS-X, Schimadzu Co., Kyoto, Japan) at a crosshead speed of $1 \mathrm{~mm} / \mathrm{min}$, until post debonding happened (Figure 1). The examples were stacked with a $1 \mathrm{~mm}$ distance cylindrical tip in an apico-coronal heading to push-out the post toward more extensive piece of the root, and cut to maintain a distance from the shape restriction. Cylindrical tip was situated to con-

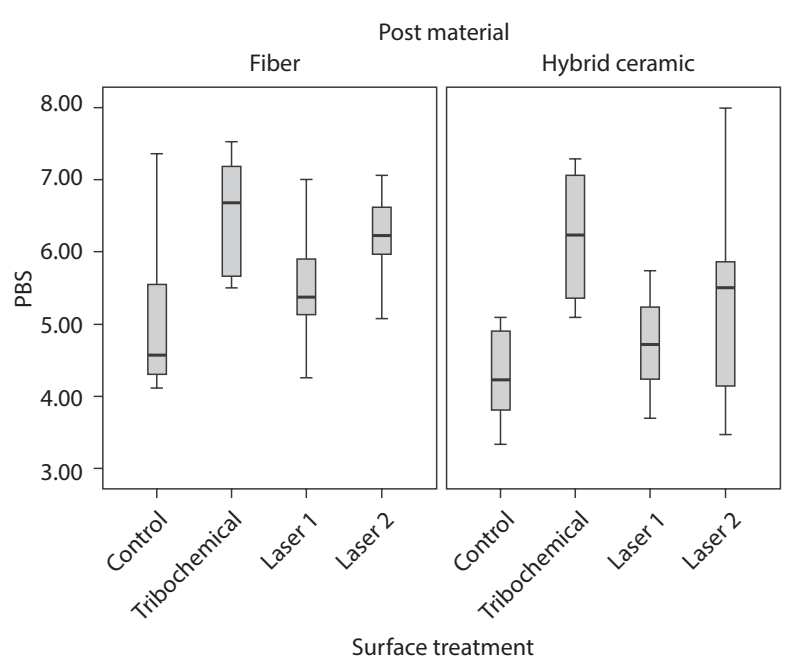

FIGURE 2. PBS values (MPa) of the test groups

tact just the post. Loads at failure were recorded as $\mathrm{N}$ and the bond strength values were determined as $\mathrm{MPa}$ by dividing $\mathrm{N}$ to the surface area.

For the statistical analysis of the data, Levene's test was used to analyze the homogeneity of variables. Two-way analysis of variance (ANOVA) and Tukey, with honestly significant differences (HSD) tests were applied for the statistical analyses of the bond strengths with a statistical software (SPSS 22.0; SPSS Inc., Chicago, IL, USA) $(\alpha=0.05)$.

\section{RESULTS}

According to two-way ANOVA, surface treatments and interactions in RNC type were statistically significant $(p<0.05)$ (Table 2$)$. The mean values and standard deviation of the test groups are presented Table 3. PBS values of the groups are showed in Figure 2.

When PBS values were examined, fiber posts demonstrated higher bonding values than RNC posts. However, these values did not exhibit statistically significant results. The highest PBS value for fiber post-groups was observed in the Tbc group (6.51 MPa). Though, this value was significantly higher than in the other groups $(p<0.05)$. Both laser applications increased PBS values compared to the control group, but there was no statistically significant difference $(p>0.05)$. The highest PBS values were observed in the Tbc group (6.22 $\mathrm{MPa})$, in the RNC post group, and this value was statistically different from the other groups $(p<0.05)$. Other surface treatments increased PBS values, but this increase was not statistically significant $(p>0.05)$.

\section{DISCUSSION}

The present study evaluated the effect of varied surfaces treatments on the PBS of two different post mate- 
TABLE 2. Variance analysis according to two-way ANOVA test

\begin{tabular}{|c|c|c|c|c|c|}
\hline Variable (source) & df & Sum of squares & Mean squares & $\boldsymbol{F}$ & $p$-value \\
\hline Post material & 9.038 & 1 & 9.038 & 10.001 & 0.002 \\
\hline Surface treatment & 29.461 & 3 & 9.820 & 10.866 & 0.000 \\
\hline Interaction & 0.997 & 3 & 0.332 & 0.368 & 0.776 \\
\hline Error & 65.071 & 72 & 0.904 & & \\
\hline Total & 2539.661 & 80 & & & \\
\hline
\end{tabular}

TABLE 3. Mean/SD (MPa) of PBS values and differences between post material/surface treatment groups

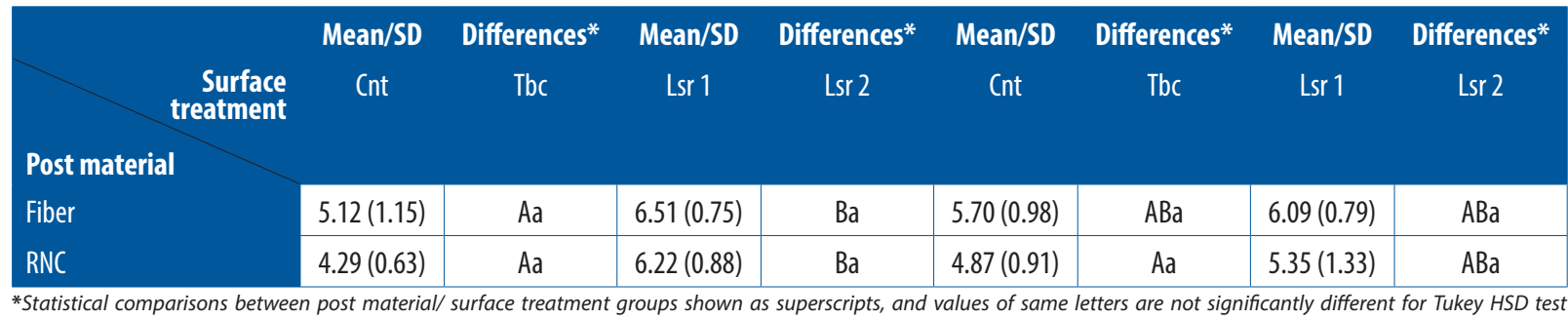
$(p>0.05)$. Superscripts with capital letters indicate differences between surface treatment groups, and small caps indicate differences between post material groups

rials. According to the results, the first null hypothesis that the bond strength to the root canal dentine would not vary depending on the type of post materials was accepted. While the PBS values were affected by the surface treatments, the second null hypothesis was rejected. The functional restoration of endodontically treated teeth is a challenge for dentists because the forces on these teeth and surrounding tissue are typically induced by internal stress [9]. In addition, due to tooth tissue loss and tooth dehydration, endodontically treated teeth are generally weak, therefore, there is a need for rehabilitation using intraradicular post system [10]. Fiber posts are widely used due to their aesthetic appearance among existing intraradicular restorations. The success of fiber post application procedures depends in part on the cementation technique between the post and the root canal dentin [11]. Zirconia, soft metal, feldspathic ceramic, and reinforced glass ceramic restorations can be fabricated by CAD/CAM procedure. These CAD/ CAM restorations have better physical and mechanical properties, higher abrasion resistance, and lesser discoloration because of the standardized industrial fabrication of the blocks. Newly developed hybrid materials called "resin-matrix ceramics" combine the physical and mechanical advantages of ceramics and improved flexural properties and low abrasiveness of composite resins. Resin nanoceramic LAVA Ultimate is one of these materials. This dental material consists of nano-ceramic particles in the resin matrix, $80 \%$ by weight. LAVA Ultimate restorations are indicated for permanent adhesive, single-unit inlay, onlays, and veneer restorations [3, 4]. Experimental evaluation of the usage of this RNC as a post material was the aim of this study.
The smoothness of the post surface limits mechanical clamping between the post and the resin core material. To form a chemical bond between methacrylate resin-based cement and post, fiber posts should react with silane molecules. For this purpose, mechanical or chemical surface treatment must be applied to the surface. In the present study, posts were sandblasted with $50 \mu \mathrm{m} \mathrm{Al}_{2} \mathrm{O}_{3}$ sands combined with silica particles as tribochemical surface treatment, and Nd-YAG laser irradiation was applied. As a result of the statistical analysis, Tbc-treated RNC and glass fiber posts indicated higher bond strength values than the control group. This result is compatible with previous studies evaluated in a systematic review [12]. It has been stated that sandblasting with Tbc can cause cracks. In previous studies, it has been proposed to reduce application time and perform a light blasting method to minimize dimensional changes in fiber posts. Therefore, as suggested, in the present study, fiber posts were blasted with Al203 particles at a pressure of 2.8 bar for 10 seconds, at a distance of $10 \mathrm{~mm}$. Mohsen evaluated the PBS of surface treatments of glass fiber and zirconia posts, and concluded that surface treatments increase bond strength for all types of posts to both root canal and resin core. As a result of the study, it can be said that sandblasting in conjunction with silica particles, followed by silane coating, is an effective surface treatment protocol for cementation of fiber posts [13].

Roughening the surface of dental materials with laser application has been proposed as a relatively reliable and simple method for surface treatment. Recent studies have evaluated the effects of Er: YAG laser application on bond strength of fiber posts in different power set- 
tings. Sipahi et al. reported that $150 \mathrm{~mJ}$ Er: YAG laser application at $10 \mathrm{~Hz}$ increased bond strength between fiber post and core material [14]. On the contrary, Tunçdemir et al. stated that Er: YAG laser applied at the same values had no effect on connection force [6]. Arslan et al. used Er: YAG laser to glass fiber posts at 150, 300, and $450 \mathrm{~mJ}$ and $10 \mathrm{~Hz}$ power settings, and concluded that $450 \mathrm{~mJ}$ Er: YAG laser application has increased the pushing and pulling force between glass fiber and resin cored material [15]. On the other hand, Kurtulmuş et al. reported on 300, 400, and $500 \mathrm{~mJ} \mathrm{Er,} \mathrm{Cr:} \mathrm{YSGG} \mathrm{laser} \mathrm{applications}$ on posts at $2 \mathrm{~Hz}$, and found that bond forces between the post and resin cores in the treated group were significantly reduced compared to the non-laser treated group. The authors stated that low bond strengths are due to high power settings damaging glass fiber [16]. The discrepancies between studies highlight not only the differences in the types of fiber post, but also the differences in the repetition rates of the applied lasers.

In a systematic review and meta-analysis, Davoudi et al. evaluated the effect of laser irradiation on push-out bond strength of dental fiber posts, and concluded that the effects of surface treatment of fiber post with laser irradiation and sandblasting with $50 \mu \mathrm{m}$ alumina might be similar as the increase of final PBS [12].

The effects of laser on dentin, ceramic, and resin composites were investigated in previous studies. However, the available data on the Nd: YAG laser effecting the fiber post surface are limited. Therefore, the aim of this present study was to investigate the effect of $\mathrm{Nd}$ : YAG laser with different power settings on micro pushout connection force between a RNC and the root wall of the fiber post. According to the statistical analysis, 1.5 and $3 \mathrm{~W}$ laser applications increased the bonding compared to the control group, regardless of post types. However, this increase was not statistically significant.

The results of this study indicated that the control group exhibited less PBS than the other groups of both post materials. In other words, surface treatments increased the connection values of the post root canal dentin. However, a statistically significant difference was observed only in the Tbc groups. This process roughened and increased irregularity of the sanded surface of the posts and the increase of the surface area, which improved the mechanical coupling for adhesive cement. The smear layer formed by preparation of post space should be eliminated according to some authors because of the increase of antibacterial effects. Furthermore, the authors indicated that the smear layer creates a weak adherent interface between the cement and dentin [17-19]. Conversely, some authors indicated not to remove the smear layer for adaptation of the materials to root canal surface $[20,21]$.

In the literature, the bond forces of posts were measured by various methods, including microtensile bond strength, push-out, and pull-out tests. The push-out test is a reliable and accurate method because it is the most appropriate test for measuring post retention. The microtensile test gives early failure. While the pull-out test uses the entire section of the root, the push-out test separates the root and allows comparison of the bond strength values in different regions of the root. In push-out tests, the load direction applied to the slices also helps to replicate clinical conditions. Thick root slices have been shown to cause irregular stress distribution, which may cause erroneous results; however, $1 \mathrm{~mm}$ thick slices can eliminate this limitation [22]. In this study, thin slices were subjected to push-out testing, and the bonding forces in the cervical regions of the root were evaluated.

The bonding process applied by a clinician has precise procedures. High level of expertise is correlated with high bond strength values. This study was carried out by researchers experienced in dental adhesions and intra-coronal posts' treatments.

As a limitation of this study, post-application surfaces were not analyzed under scanning electron microscopy (SEM). In further research, it would be useful to use SEM to evaluate the effect of surface treatments on post surfaces.

\section{CONCLUSIONS}

The experimentally produced RNC posts showed PBS values close to the prefabricated fiber posts. However, it is thought that the bonding values would be higher, as the connection surface area of the RNC posts can be prepared as a single piece with crown. Therefore, this issue should be considered in future studies, and working methodology should be created under the above-mentioned circumstances.

\section{ACKNOWLEDGEMENT}

This in vitro study has been financially supported by Ordu University Department of Scientific Investigation Projects, No. AR-1524.

\section{CONFLICT OF INTEREST}

The authors declare no potential conflicts of interest with respect to the research, authorship, and/or publication of this article.

\section{References}

1. Martin F, Ozcan M. Adhesion of bulk-fill resin composites as core and intraradicular post materials only versus the use of glassfiber posts in different regions of root dentin. Journal of Adhesion Science and Technology 2019; 33: 1410-1425.

2. Fredriksson M, Astbäck J, Pamenius M, Arvidson K. A retrospective study of 236 patients with teeth restored by carbon fiber-reinforced epoxy resin posts. J Prosthet Dent 1998; 80: 151-157.

3. Çelik E, Şahin SC, Dede DÖ. Effect of surface treatments on the bond strength of indirect resin composite to resin matrix ceramics. J Adv Prosthodont 2019; 11: 223-231. 
4. Çelik E, Şahin SC, Dede DÖ. Shear bond strength of nanohybrid composite to the resin matrix ceramics after different surface treatments. Photomed Laser Surg 2018; 36: 424-430.

5. Giardino L, Del Fabbro M, Cesario F, Fernandes FS, Andrade FB. Antimicrobial effectiveness of oxidant and chelating agents combination in infected dentine: an ex vivo confocal laser scanning microscopy study. Int Endod J 2018; 51: 448-456.

6. Tuncdemir AR, Yildirim C, Ozcan E, Polat S. The effect of a diode laser and traditional irrigants on the bond strength of self-adhesive cement. J Adv Prosthodont 2013; 5: 457-463.

7. Angeloni V, Mazzoni A, Marchesi G, et al. Role of chlorhexidine on long-term bond strength of self-adhesive composite cements to intraradicular dentin. J Adhes Dent 2017; 19: 341-348.

8. Çakııı F, Çakııı EB, Ceyhanlı KT, Celik E, Kucukekencı FF, Gunseren AO. Evaluation of bond strength of various epoxy resin based sealers in oval shaped root canals. BMC Oral Health 2016; 16: 106.

9. Kahn FH, Rosenberg PA, Schulman A, Pines M. Comparison of fatigue for three prefabricated threaded post systems. J Prosthet Dent 1996; 75: 48-53.

10. Bitter K, Noetzel J, Volk C, Neumann K, Kielbassa AM. Bond strength of fiber posts after the application of erbium:yttriumaluminum-garnet laser treatment and gaseous ozone to the root canal. J Endod 2008; 34: 306-309.

11. Uzun I, Keskin C, Özsu D, Güler B, Aydemir H. Push-out bond strength of oval versus circular fiber posts irradiated by erbiumdoped yttrium aluminum garnet laser. J Prosthet Dent 2016; 116: 425-430.

12. Davoudi A, Mosharraf R, Akhavan A, Zarei F, Pourarz S, Iravani S. Effect of laser irradiation on push-out bond strength of dental fiber posts to composite resin core buildups: a systematic review and meta-analysis. Photodiagnosis Photodyn Ther 2019; 27: 184-192.

13. Mohsen CA. Evaluation of push-out bond strength of surface treatments of two esthetic posts. Indian J Dent Res 2012; 23: 596-602.

14. Sipahi C, Piskin B, Akin GE, Bektas OO, Akin H. Adhesion between glass fiber posts and resin cement: evaluation of bond strength after various pre-treatments. Acta Odontol Scand 2014 72: 509-515

15. Arslan H, Kurklu D, Ayrancı LB, et al. Effects of post surface treatments including Er:YAG laser with different parameters on the pull-out bond strength of the fiber posts. Lasers Med Sci 2014; 29: 1569-1574.

16. Kurtulmus-Yilmaz S, Cengiz E, Ozan O, Ramoğlu S, Yılmaz HG. The effect of Er,Cr:YSGG laser application on the micropushout bond strength of fiber posts to resin core material. Photomed Laser Surg 2014; 32: 574-581.

17. Bystrom A, Claesson R, Sundqvist G. The antibacterial effect of camphorated paramonochlorophenol, camphorated phenol and calcium hydroxide in the treatment of infected root canals. Endod Dent Traumatol 1985; 1: 170-175.

18. Perez F, Calas P, de Falguerolles A, Maurette A. Migration of a Streptococcus sanguis strain through the root dentinal tubules. J Endod 1993; 19: 297-301.

19. Kennedy WA, Walker WA 3rd, Gough RW. Smear layer removal effects on apical leakage. J Endod 1986; 12: 21-27.

20. Gençoğlu N, Samani S, Günday M. Dentinal wall adaptation of thermoplasticized gutta-percha in the absence or presence of smear layer: a scanning electron microscopic study. J Endod 1993; 19: 558-562.

21. Gutmann JL. Adaptation of injected thermoplasticized guttapercha in the absence of the dentinal smear layer. Int Endod J 1993; 26: 87-92.

22. Otani A, Amaral M, May LG, Cesar PF, Valandro LF. A critical evaluation of bond strength tests for the assessment of bonding to Y-TZP. Dent Mater 2015; 31: 648-656. 